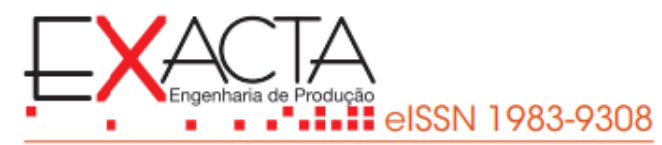

https://doi.org/10.5585/ExactaEP.v18n3.8473

\title{
Aplicação do Jidoka em um processo SMT: estudo de caso
}

\author{
Jidoka application at a SMT process: case study
}

\author{
Iracyanne Retto Uhlmann ${ }^{1}$ \\ (iD) Carlos Américo de Souza Silva ${ }^{2}$ \\ Djonathan Luiz de Oliveira Quadras ${ }^{3}$ \\ (iD) Enzo Morosini Frazzon ${ }^{4}$
}

${ }^{1}$ Doutora em Engenharia de Produção pela Universidade Federal de Santa Catarina iracyanne.uhlmann@gmail.com

2 Universidade Federal de Santa Catarina. Doutorando em Engenharia de Produção. Florianópolis/SC, Brasil. camericoss@gmail.com

${ }^{3}$ Universidade Federal de Santa Catarina. Graduando em Engenharia Elétrica. Florianópolis/SC, Brasil. oliveira.ind.eng@gmail.com

${ }^{4}$ Universidade Federal de Santa Catarina. Professor Associado do Departamento de Engenharia de Produção e Sistemas. Florianópolis/SC, Brasil. enzo.frazzon@ufsc.br

\section{Resumo}

Produção com qualidade é assunto crítico em qualquer processo, mais especialmente em processos cujo recurso predominante seja o uso de máquinas, as quais caso não estejam ajustadas ou capacitadas a detectar falhas, podem contribuir para altos custos de desperdício de defeitos em série, seja pelo reprocesso ou pelo refugo de produtos não conformes. O objetivo deste artigo é apresentar um estudo de caso na aplicação do Jidoka em um processo de Surface Mount Technology (SMT), essa ferramenta foi usada para direcionar ações que contribuíram na detecção e eliminação de defeitos de set top box sem vídeo. A pesquisa foi aplicada em uma empresa multinacional do Polo Industrial de Manaus (PIM). A metodologia foi guiada pelo Define, Measure, Analyze, Improve and Control (DMAIC). Como resultados, a ferramenta se mostra adequada para este tipo de processo, ganho financeiro foi evidenciado, além da redução significativa de problemas no produto final.

Palavras-chave: Jidoka. Qualidade. SMT. Manufatura Enxuta. DMAIC.

\section{Abstract}

Production with quality is a critical issue in any process, especially in processes whose predominant feature is the use of machines, which if not adjusted or capable of detecting failures, can contribute to high costs of defect waste, either through rework or scrap of nonconforming products. The objective of this article is to present a case study of Jidoka application in a Surface Mount Technology (SMT) process, this tool was used to drive actions that contributed to the detection and elimination of no video defect in set top box. The research was applied in a multinational company from Manaus Industrial Pole (PIM). The methodology was guided by Define, Measure, Analyze, Improve and Control (DMAIC). As results, the tool is suitable for this type of process, financial gain was evident, besides the significant reduction of problems in the final product.

Keywords: Jidoka. Quality. SMT. Lean Manufacturing. DMAIC. 
1 Introdução

O avanço tecnológico gera no cenário industrial uma corrida pela rápida adaptação às novas tecnologias e pelo desenvolvimento de processos robustos, competitivos e com menos riscos de falhas (Oliveira, Santos, Mazzuco \& Frazzon, 2017). Buscando a efetividade de suas operações, empresas do mundo todo têm buscado a implementação do Lean Manufacturing (LM) em seus modelos de produção (Uhlmann, Silva, Oliveira, \& Frazzon, 2017)

Segundo Soliman e Saurin (2017) e Jasti e Kodali (2014), o LM é comumente conhecido como uma filosofia para melhorar a performance de sistemas de produção por meio da eliminação de desperdícios e pela entrega de valor aos clientes. A utilização do LM se mostra relevante por sua aplicação não demandar a necessidade de grandes investimentos financeiros e soluções complexas, pois mudanças pequenas e simples podem gerar grandes resultados.

O surgimento dessa filosofia remete ao Toyota Production System (TPS) (Iwao, 2017 ; Nunes, Vaccaro, \& Antunes Júnior, 2017). Um dos principais problemas enfrentados por Sakichi Toyoda, fundador da Toyota, foi que qualquer máquina que funcione de forma automática não interrompe suas atividades quando uma situação anormal ocorre, o que leva ao acúmulo de peças com defeitos. A fim de eliminar este risco, devido ao aumento da sua própria produção, a Toyota criou o conceito "Jidoka", o qual significa "automação com espírito humano", sendo traduzido como auto ativação, e amplamente aceito na literatura como autonomação, ou seja, a automação sendo utilizada em conjunto com o trabalho humano. Segundo Taiichi Ohno, "O Jidoka dá inteligência para a máquina." (Audenino, 2012 ; Womack, Jones, \& Roos, 2007).

Essa ferramenta tem sido utilizada largamente em vários tipos de processos, e consequentemente, vários estudos de caso têm sido publicados sobre esse assunto. Araujo e Costa (2012) e Araujo (2012) caracterizaram seu uso em um ambiente de alta complexidade e diversificação em termos de consumo de itens no âmbito hospitalar, em seu trabalho comparou-se melhorias da redução de erros de dispensação e movimentação de materiais e medicamentos liberados pelo serviço antes e depois das alterações, confirmando-se melhoria sistêmica de toda a cadeia produtiva, maximizando os recursos geradores de riquezas através da identificação e do gerenciamento dos materiais e medicamentos utilizados pela unidade hospitalar. Ferreira e Ciuccio (2012) propuseram-se a explorar o conceito em relação a capacidade de se ajustar às grandes variações de demanda e o melhor aproveitamento do capital intelectual da empresa, como conclusão, analisaram que a autonomação proporciona às empresas que a adotam uma maior flexibilidade para casos de variação de demanda, visto que um mesmo operador pode acompanhar a operação de um número maior de máquinas, pois estas estarão dotadas de sistemas de controle que as impedem de propagar erros, então a capacidade gerada através da adoção da autonomação poderá ser aproveitada, quando em excedente de tempo, com a aquisição de novas máquinas que poderão ser atendidas pelo mesmo operador, pois diminui o risco de dependência operador-máquina. Também há estudos sobre o uso do Jidoka junto 
com a utilização de andons para auxílio da melhoria aos impactos causados por downtimes numa fábrica de computadores (Bove \& Gerber, 2013); na indústria de componentes de refrigeração (Guimaraes, Colares, Medeiros, \& Santana, 2014) e na criação de um indicador remoto de falhas como ferramenta de auxílio à autonomação (Moura, 2014). Silva e Santos (2010) atestaram a ferramenta Jidoka como parte intrínseca da construção de máquinas de processo Surface Mount Technology (SMT). Entretanto, defeitos ocorrem também nesse tipo de processo, então qual outra maneira de aplicar o Jidoka para resolver falhas em processo de SMT em um ambiente real?

Nesta pesquisa, os próprios autores confirmam o fato do assunto autonomação não ser muito abordado na literatura, tanto para tratamento teórico como para os práticos. O objetivo do artigo é de apresentar um estudo de caso sobre a aplicação da ferramenta Lean Jidoka em um processo de SMT, o qual contribuiu para eliminar defeitos de set top boxes sem vídeo causados por falha de coplanaridade no conector HDMI em uma multinacional de eletroeletrônicos do Polo Industrial de Manaus (PIM). A contribuição e relevância deste trabalho podem ser evidenciadas pela multiplicação dos conceitos de Jidoka na busca de solução de outros problemas de qualidade em outras linhas de SMT e também Inserção Manual (IM) da mesma fábrica, sendo este o reconhecimento de todos os colaboradores e gestores. Sendo assim, atesta-se que este estudo pode ser aplicado em outras organizações industriais.

\section{Referencial Teórico}

Nesta seção será apresentada a revisão teórica acerca do LM e Six Sigma e algumas de suas ferramentas relevantes aos objetivos do estudo.

\subsection{Lean Manufacturing}

Originário da Toyota Motor Corporation, o LM é considerado como uma alternativa radical para o método tradicional de produção em massa e um conjunto de princípios para maximizar eficiência operacional, qualidade, velocidade e custo (Holweg, 2007).

De acordo com (Radnor, Holweg, \& Waring, 2012) embora conceitualmente simples, não é fácil definir o LM. O núcleo da filosofia é melhorar continuamente o processo por meio da remoção de valores não agregados ou desperdícios. Porém, o foco somente sobre desperdício restringe o escopo do LM, dado que o foco em desperdícios é somente um dos três conceitos inter-relacionados. O LM pode ser definido como uma prática de gerenciamento baseada na filosofia de processos de melhoria contínua, seja pelo aumento do valor ao cliente ou pela redução de atividades de valor não agregado, variações no processo e condições de trabalho inadequadas.

A fim de ilustrar melhor os fundamentos do LM, algumas ferramentas foram organizadas em um esquema chamado "A casa do TPS", conforme mostrado na Figura 1. 


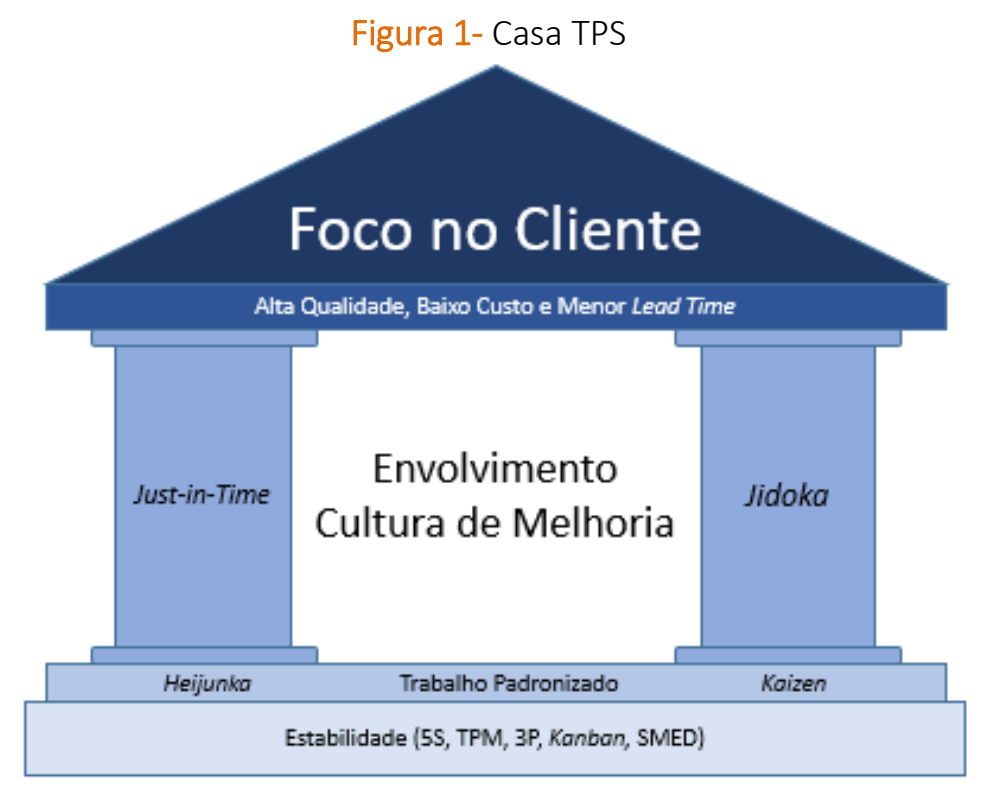

Fonte: Adaptada de Gonçalves (2009).

Como base que sustenta a casa temos a estabilidade da fábrica, surgindo ferramentas como os 5 sensos (5S), Total Productive Maintenance (TPM), os 3Ps, Kanban, Single Minute Exchange of Die (SMED). Os dois pilares base para o enfoque no cliente são o Just-in-Time (JIT), com fluxos contínuos e Produção Puxada; enquanto o Jidoka visa a construção da qualidade. Por fim, o foco no cliente surge com os objetivos de alcançar uma maior qualidade a um baixo custo e menor Lead Time.

\subsubsection{Jidoka}

Jidoka é a capacidade das linhas de produção serem paradas, eventualmente, quando os problemas ocorrerem, como: mal funcionamento do equipamento, problemas de qualidade ou de trabalho atrasado; quer seja por máquinas que têm a capacidade de detectar anormalidades ou pelos funcionários. Visa prevenir produção de quaisquer produtos defeituosos com a parada da linha se houver um caso de irregularidade, então somente produtos sem defeitos são enviados para o processo seguinte. Por isso, a qualidade deve ser construída em cada processo dos produtos e não há necessidade de dedicar operadores apenas para monitorar as máquinas (economia de mão de obra) (Abdulmouti, 2015).

As etapas básicas de Jidoka são: (1) detectar o problema, (2) parar o processo, (3) restaurar o processo para a função adequada, (4) investigar a causa raiz do problema, e (5) instalar contramedidas. Cada trabalhador da Toyota tem o poder de parar a linha de montagem. De um modo geral, parar linha de montagem é muito caro e é normalmente evitado. Inicialmente, as linhas onde os trabalhadores podem parar o processo apresentam menor produção. Como paradas levam para resolução de problemas, com o passar do tempo a linha terá menos interrupções e melhor qualidade em comparação com uma linha em que os trabalhadores não têm o poder de criar paralisações (Grout \& Toussaint, 2010). 


\subsubsection{Poka-Yoke}

Ainda nesse contexto de Jidoka, é muito apropriado falarmos sobre poka-yoke. Uma variedade de opiniões sobre esses termos poderia levar alguém a pensar que poka-yoke é um subconjunto do Jidoka, ou vice-versa. Jidoka e poka-yoke estão relacionados com parar o processo. Jidoka para a linha, a fim de resolver os problemas. Poka-yoke interrompe o processo, a fim de restaurar o processo aos seus parâmetros de funcionamento apropriados, ou para remover as causas de defeitos. Poka-Yoke pode ser uma das medidas tomadas em resposta a problemas que surgiram com o Jidoka (Grout \& Toussaint, 2010).

Poka-yoke é uma gíria japonesa mais frequentemente traduzida como "à prova de erros". Poka significa erros involuntários. Yoke é uma forma de yokeru, que significa evitar (Shingo, 1986).

Tsuda (1993) criou uma tipologia alternativa para "à prova de erros" que foi dividida nas seguintes categorias: prevenção de erros, deteç̧ão de erros, prevenção da influência de erros, e à prova de erros no ambiente de trabalho. Prevenção de erro é a forma mais forte de à prova de erros, ela evita a ocorrência de erros. Detecção de erros é apenas um alerta aos trabalhadores sobre um erro já ocorrido. Prevenir a influência de erros significa mitigar o impacto dos erros já ocorridos. Prevenção de erros no ambiente de trabalho significa reduzir a desordem, confusão e ambiguidade onde o trabalho é feito.

A diferenciação entre Jidoka e Poka-Yoke pode ser pouco confusa. Em um processo de produção, o Jidoka irá paralisar todas as atividades (seja em uma máquina apenas, ou, se necessário, em todo o processo) ao surgir uma situação anormal que vá levar ao erro, enquanto o Poka-Yoke são as ações tomadas antes, buscando evitar que ocorra o erro.

\subsubsection{Andon}

Andon é um indicador visual de parada de linha, informando a localização e a natureza da situação problemática em um passar de olhos (Ohno, 1988). É um sinalizador que busca informar quando uma anormalidade ocorreu.

Andons podem ser utilizados para controlar a qualidade da produção e melhorar os processos de detecção de defeitos. Usam um alarme para alertar os trabalhadores quando há uma alteração no status. Quando um alarme é ativado, ele diretamente indica um problema em uma linha de produção particular. Um funcionário pode parar a linha de produção e chamar por ajuda para ajustar a máquina ou consertar um defeito de qualidade (Li \& Blumenfeld, 2007).

\subsection{Six Sigma}

“O Six Sigma teve início na década de 1980 na Motorola, mas ganhou notoriedade em 1996 quando a General Eletric por meio de seu CEO Jack Welch anunciou um resultado de 1,5 bilhão de 
dólares de redução de custo por meio deste programa" (Junior \& Calarge, 2013). Ele tem sido considerado um poderoso método de gerenciamento que emprega uma metodologia de melhoria contínua bem estruturada para reduzir a variabilidade e os defeitos de processos usando ferramentas e técnicas estatísticas eficazes, sendo também uma estratégia que se concentra em melhorar o entendimento dos requisitos do cliente, as estratégias da organização, a produtividade e a qualidade dos processos e o desempenho financeiro (Wei \& Cheng, 2018).

\subsubsection{DMAIC}

Não importa qual seja a abordagem para implantar melhorias dentro da empresa, ter um modelo de melhoria padrão como DMAIC é extremamente útil, pois fornece à empresa um roteiro de melhoria (Wang \& Chen, 2010). Esta abordagem é uma ferramenta que funciona como um filtro para passar de um problema complexo com variáveis não controladas para uma situação onde a qualidade é controlada. Este método segue cinco passos necessários para obter resultados confiáveis: Definir, Medir, Analisar, Melhorar (ou Inovar) e Controlar (ou Checar) (Al-bahi \& Soliman, 2018 ; Dahlgaard \& Dahlgaard-Park, 2006 ; Pyzdek \& Keller, 2014 ; Youssouf, Rachid, \& Ion, 2014). 0 Quadro 1 explica cada uma das atividades:

\section{Quadro 1 - Visão Geral do DMAIC}

Definir os objetivos da atividade de melhoria e incorporá-los em um Termo de Abertura do Projeto. Obter patrocínio e reunir a equipe.

Medir o sistema existente. Estabelecer indicadores válidos e confiáveis para ajudar a monitorar o progresso em direção aos objetivos definidos na etapa anterior.

A Analisar o sistema para identificar formas de eliminar a lacuna entre o desempenho atual do sistema ou processo e o objetivo desejado.

Melhorar o sistema. Seja criativo para encontrar novas maneiras de fazer com que as coisas fiquem melhores, mais baratas ou mais rápidas.

c Controlar o novo sistema. Institucionalize o sistema melhorado através da modificação dos sistemas de recompensa e incentivo, procedimentos, instruções de operação e outros sistemas de gerenciamento.

Fonte: Adaptado de Pyzdek e Keller (2014).

\subsection{Lean Six Sigma}

"O Lean Six Sigma surgiu da união de duas abordagens de gestão conhecidas como Lean Production, desenvolvida com base no TPS, e o Six Sigma desenvolvido pela empresa Motorola" (Junior \& Calarge, 2013). As empresas, de modo geral, têm usado o Lean Six Sigma (LSS) para enfatizar o processo de melhoria de qualidade e serviço oferecido pelo Six Sigma e as ferramentas de redução de custos e produtividade oferecidas pela gestão Lean (Wang \& Chen, 2010). De acordo com Uhlmann (2015), esta combinação é bem apropriada, pois é comum encontramos em vários processos produtivos, a aplicação de ferramentas do Lean (Jidoka, SMED, TPM, etc.), orientadas pelo uso de ferramentas do six sigma (A3 e DMAIC), a exemplo dos estudos de Alvarez (2015), Chen e Lyu (2009), 
Kumar et al. (2006), Vinodh, Kumar e Vimal (2014) e Wang e Chen (2010), os quais aplicaram a abordagem DMAIC.

\section{Metodologia}

Muitos dos conceitos inovadores e das teorias no gerenciamento de operações, desde produção Lean até estratégia de manufatura, têm sido desenvolvidos por meio de pesquisa de caso em campo, enriquecendo não somente a teoria, mas também os próprios pesquisadores (Voss, Tsikriktsis, \& Frohlich, 2002).

De acordo com Yin (2015): "O estudo de caso é a estratégia escolhida ao se examinarem acontecimentos contemporâneos, mas quando não se podem manipular comportamentos relevantes, [...] acrescenta duas fontes de evidências [...]: observação direta dos acontecimentos que estão sendo estudados e entrevistas das pessoas neles envolvidas".

Muitas pesquisas utilizam o método de melhoria contínua do Six Sigma, inclusive direcionado pelo DMAIC, como os estudos de Kumar et al. (2006) e Vinodh et al. (2014).

A partir de um problema real de qualidade que estava afetando o cliente, representantes de diversas áreas (qualidade de processo, qualidade de materiais, produção, engenharia e compras) reuniram-se para analisar e encontrar uma solução. A coleta de dados aconteceu por meio da observação direta dos acontecimentos e entrevistas com os funcionários envolvidos, além das informações contidas nos indicadores da qualidade. Para tratar o problema complexo foi decido usar o DMAIC para organizar as ideias e chegar a soluções que recuperassem a satisfação do cliente.

Esta pesquisa foi aplicada em uma empresa multinacional fabricante de produtos eletroeletrônicos no setor industrial de serviços de manufatura, a qual disponibiliza para seus clientes um completo leque de serviços, incluindo os de elaboração de projetos, gerenciamento de materiais, fabricação, execução de testes, montagem de sistemas e pronto atendimento de seus pedidos, oferecendo para cada um, soluções sob medida com a melhor qualidade possível.

Sua filial, no PIM, desenvolve as atividades de montagem de conjuntos e subconjuntos para set top box, câmeras fotográficas, placas de TV LED e leitoras para cartão de crédito. Adota o modelo de Unidade de Negócio, sendo que cada unidade fornece pessoal dedicado e recursos focados nas necessidades individuais de cada cliente e de suas linhas de produção, desta forma, tanto os maiores quantos os menores clientes são tratados com a mesma dedicação, como se existisse uma mini fábrica dentro da empresa.

Escolheu-se o processo de SMT da unidade de negócio que produz placas de set top box, mais especificamente a linha do modelo Alfa, por ser o carro chefe dessa célula e ter sido alvo de reclamação direta sobre falhas de produtos sem vídeo ocorridas nas instalações do cliente externo, ou seja, após a venda do produto. 


\section{Estudo de Caso}

\subsection{Fase 1: Definir}

\subsubsection{Definição do Time}

Seria impossível desenvolver um projeto como este sem o envolvimento de pessoas, gente é o principal recurso para aplicação de qualquer uma das ferramentas Lean (Uhlmann, 2015). Desse modo, para facilitar a condução desse evento, foi montado um comitê com representantes de todos os departamentos: coordenação de LM, Manufatura, Engenharia da Qualidade, Engenharia de Teste, Engenharia Industrial, Engenharia de Manufatura, Planejamento, Compras e Almoxarifado, além da participação dos funcionários diretos da linha (operadores, técnicos, líderes, revisores e inspetores).

Este evento Jidoka foi liderado pelo Engenheiro de Qualidade. Todos os membros do comitê foram desafiados a encontrar solução para eliminar um defeito epidêmico ocorrido no cliente. A construção da qualidade foi aplicada por todos tornando o processo autossuficiente na detecção, e as ações corretivas foram implementadas, eliminando a causa raiz.

\subsubsection{Definição do Problema}

O Comitê Lean foi chamado pela gerência da célula para ajudar a resolver um problema epidêmico que estava deixando o cliente insatisfeito, produtos sem vídeo causado por problema de coplanaridade no componente HDMI, posição SK9600, que é mostrado na Figura 2. O desafio de meta lançado ao time: zero defeito em todos os processos da empresa e no cliente, tendo como consequência satisfação do cliente e economia com reparos.

Figura 2 - Reclamação oficial recebida do cliente

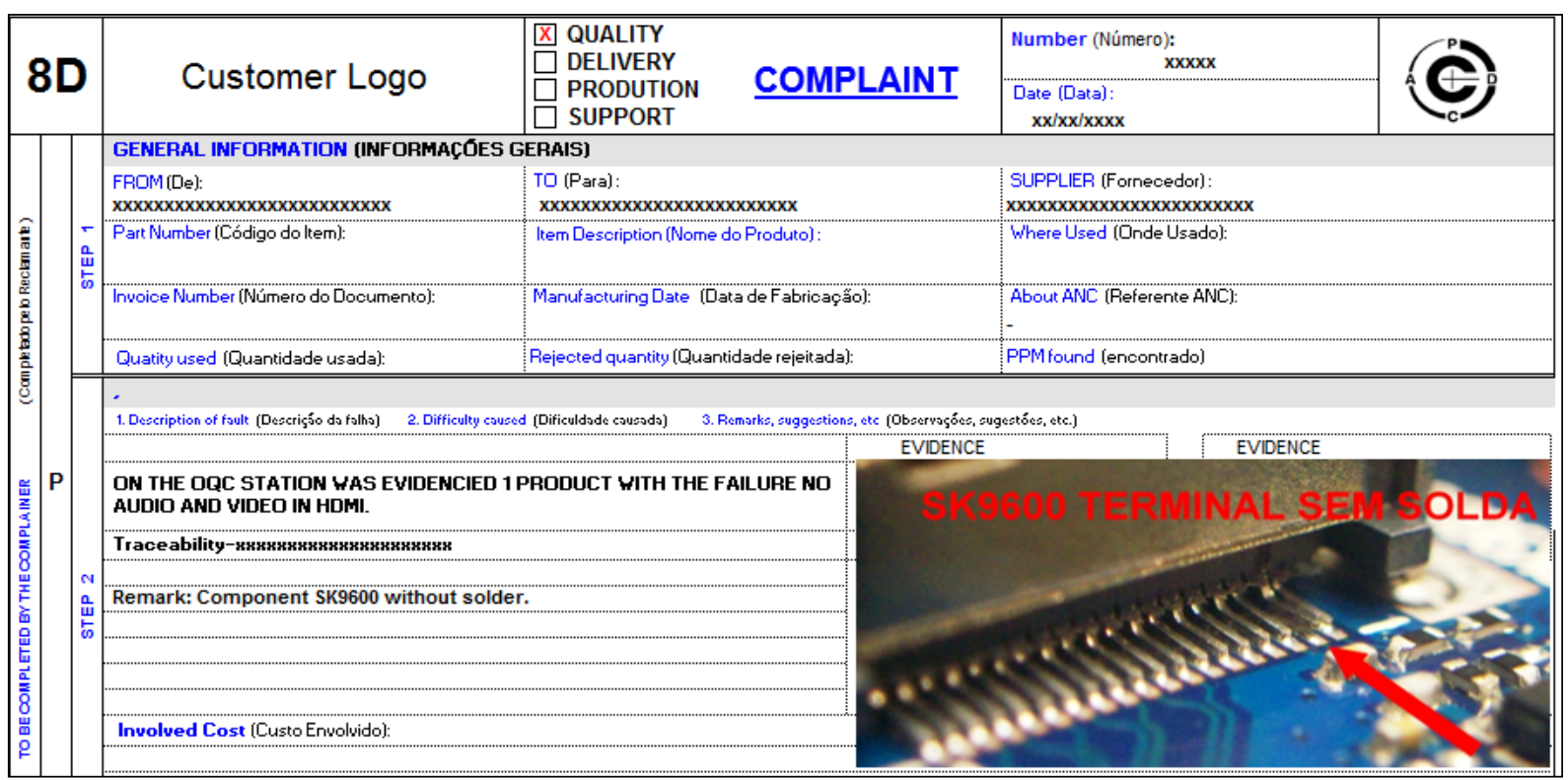

Fonte: Cliente da empresa estudada. 


\subsection{Fase 2: Medir}

O time coletou e consolidou os dados de defeitos de coplanaridade no HDMI, em todas as fases do processo (SMT, inserção manual e cliente final) a fim de obter a informação necessária para confirmar o defeito e comparar entre os diversos pontos de medição onde o problema estava ocorrendo. Era necessário investigar se havia algum filtro de detecção falhando.

Os dados do cliente final (cliente externo) foram registrados a partir das informações recebidas. Os dados iniciais do processo de SMT (origem) foram coletados a partir dos formulários com registros manuais realizados pelos operadores de inspeção visual das placas. Já os dados da inserção manual (cliente interno) foram levantados automaticamente pelos testes funcionais, a partir de suas instalações.

Conforme ilustrado na Figura 3, o impacto desse defeito no cliente externo começou na semana 07. A empresa recebeu uma reclamação formal sobre o alto índice de produtos sem vídeo.

Figura 3 - Defeitos no cliente externo

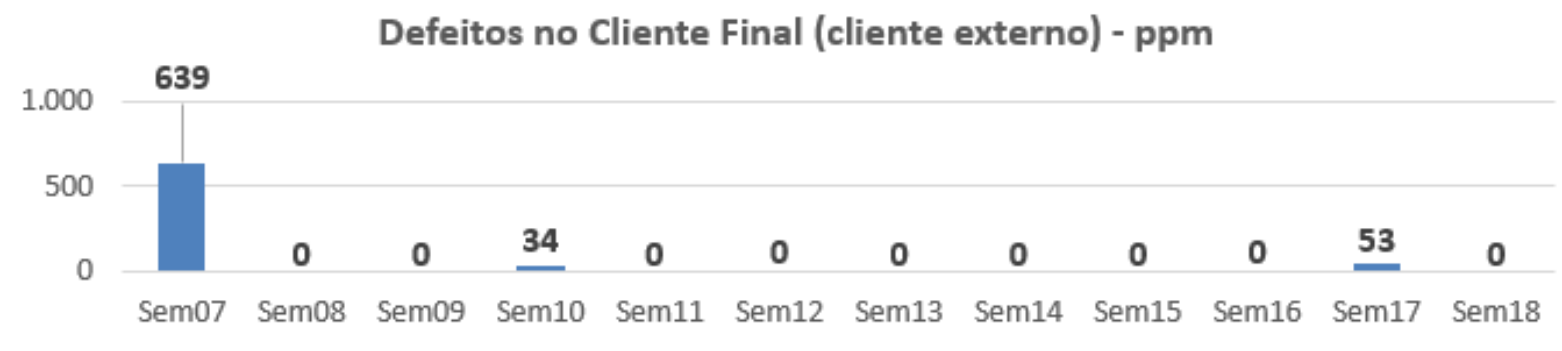

Fonte: Autores.

Como ação de contenção imediata, após a reclamação, a linha de SMT orientou os operadores responsáveis pela inspeção visual a darem foco na procura de defeitos de coplanaridade do componente SK9600, esta ação aumentou a detecção na linha de SMT a partir da semana 08 (Figura 4) e reduziu os impactos na linha de inserção manual (Figura 5) e, mitigou o sintoma no cliente final (Figura 3), porém foi só uma disposição de detecção, o problema ainda continuava a ocorrer.

Figura 4 - Defeitos na origem

\section{Defeitos na SMT (origem) - ppm}

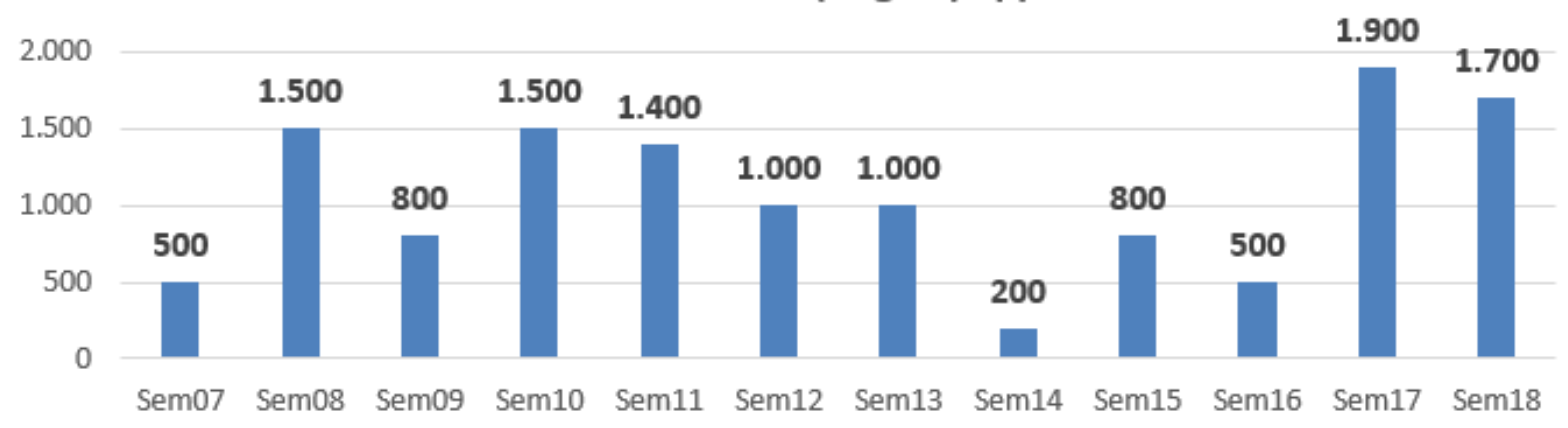

Fonte: Autores. 
Na semana 09 o time de engenharia de teste adicionou teste funcional por amostragem para detectar o problema na saída da linha de inserção manual (cliente interno) a fim de evitar que o defeito chegasse até o cliente externo. A informação é mostrada na Figura 5:

Figura 5 - Defeitos no cliente interno

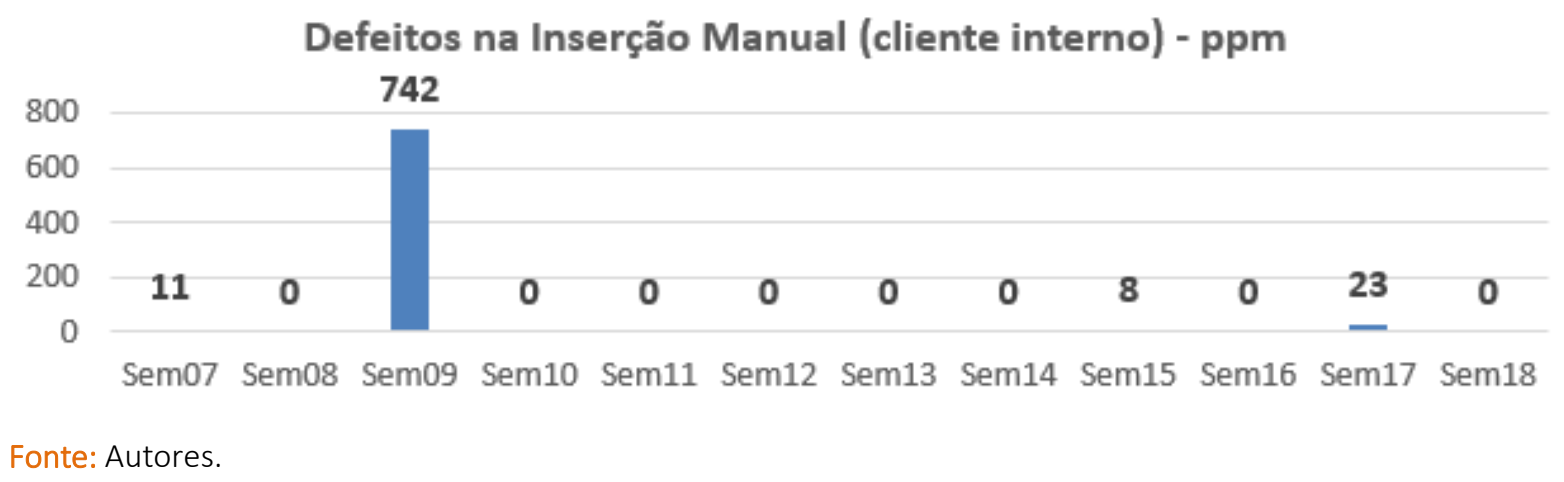

\subsection{Fase 3: Analisar}

O time multifuncional se reuniu e por meio de brainstorming listou doze possíveis causas para o problema. Com ajuda da ferramenta Cause \& Effect Matrix (CE Matrix) cada membro (nomeados neste estudo de $A$ à $G$ ) pontuou (escala de 1 à 9 , sendo 1 menos significativo e 9 mais significativo) o quanto acreditava que determinada causa possível era de fato a causa real para o problema de produtos sem vídeo no cliente. Multiplicando a pontuação de todos os membros, o item "componente com terminal desalinhado" teve o maior resultado, conforme ilustrado no Quadro 2. Dessa forma, o time concluiu que componente HDMI com coplanaridade era a causa raiz do defeito.

\section{Quadro 2-CE Matrix}

\begin{tabular}{|l|c|c|c|c|c|c|c|c|}
\hline Causas Possíveis & A & B & C & D & E & F & G & Resultado \\
\hline Falha no feeder & 1 & 1 & 1 & 1 & 1 & 9 & 1 & 9 \\
\hline Etiqueta de rastreabilidade mal posicionada & 3 & 1 & 1 & 1 & 1 & 1 & 1 & 3 \\
\hline Inspeção visual não detecta 100\% das falhas & 1 & 3 & 1 & 1 & 1 & 1 & 3 & 9 \\
\hline Reuso do material que foi rejeitado pela máquina & 3 & 1 & 1 & 1 & 3 & 1 & 1 & 9 \\
\hline Feeder não calibrado & 1 & 1 & 1 & 1 & 1 & 3 & 1 & 3 \\
\hline Nozzle quebrado & 3 & 1 & 1 & 1 & 1 & 9 & 1 & 27 \\
\hline Falha na transferência do componente & 1 & 1 & 1 & 1 & 1 & 9 & 1 & 9 \\
\hline Pasta de solda deslocada & 1 & 1 & 1 & 1 & 1 & 9 & 1 & 9 \\
\hline Solda insuficiente & 3 & 1 & 1 & 1 & 1 & 9 & 1 & 27 \\
\hline Componente com terminal desalinhado & 9 & 9 & 9 & 9 & 9 & 9 & 9 & 4.782 .969 \\
\hline Material com dimensões fora do especificado & 1 & 1 & 1 & 1 & 1 & 1 & 1 & 1 \\
\hline Fita do carretel com adesivo em excesso & 1 & 1 & 1 & 1 & 1 & 3 & 1 & 3 \\
\hline
\end{tabular}

Fonte: Autores.

A fim de validar sua conclusão e tomar ações definitivas para eliminar a causa raiz do problema, o time inspecionou visualmente por amostragem o componente ainda dentro do casulo do carretel e encontrou amostras com defeito. Foi, então, enviado um relatório com reclamação formal ao 
fornecedor (Figura 6):

Figura 6 - Reclamação oficial enviada ao fornecedor

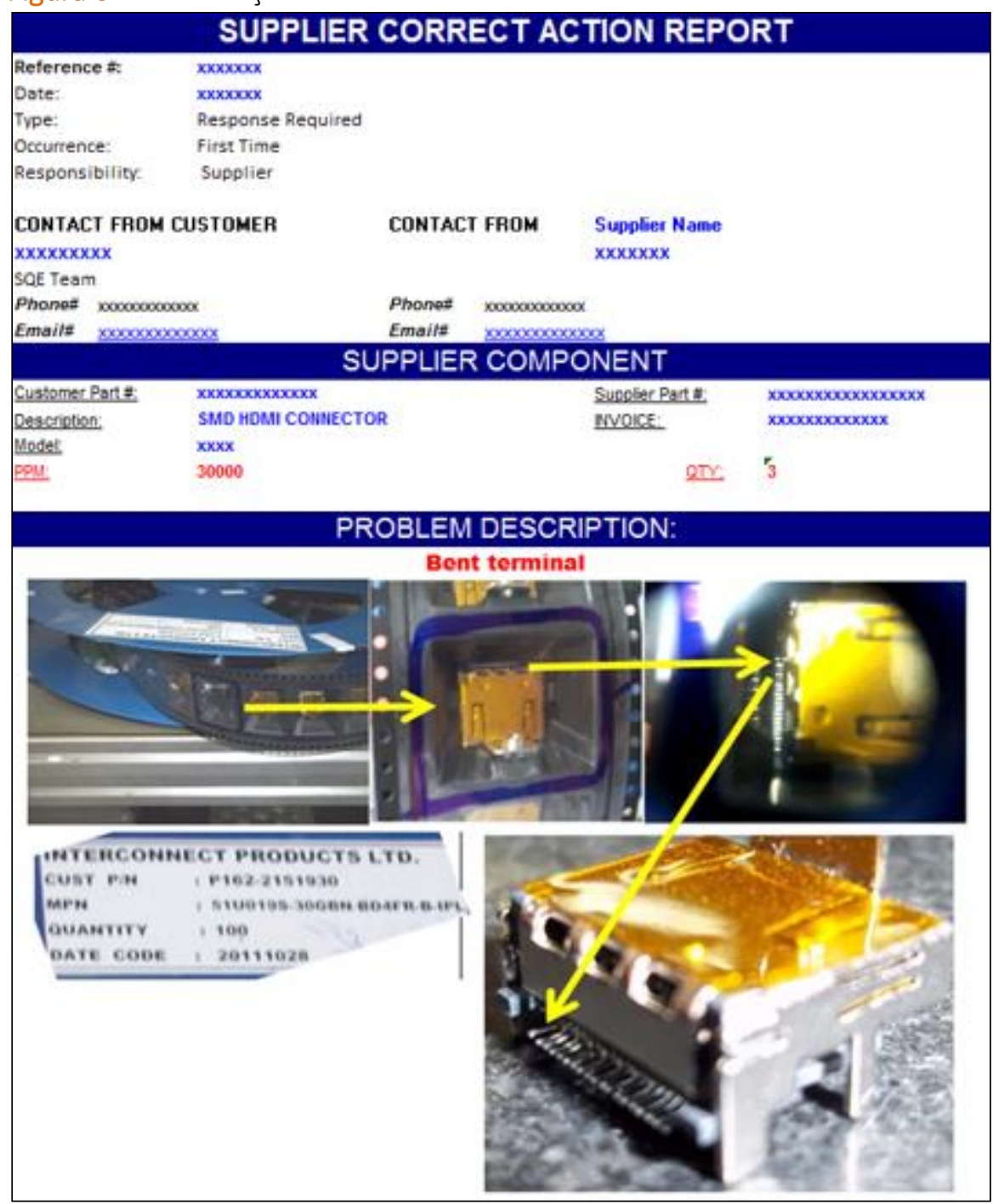

Fonte: Empresa estudada.

\subsection{Fase 4: Melhorar}

Como resultados da aplicação da metodologia tivemos as ações para melhorias imediatas:

- Semana 08: orientação aos operadores de inspeção visual da linha de SMT (origem) a darem foco 100\% na procura de defeitos de coplanaridade do componente SK9600;

- Semana 09: inclusão de teste funcional, por amostragem, na linha de inserção manual (cliente interno), para detecção do sintoma sem vídeo;

- Semana 10: inclusão de Automatic Optical Inspection (AOI) para inspeção ótica automática na linha de SMT (origem), esta máquina interrompe as atividades sempre que a falha for detectada, funcionando como Poka-Yoke e Andon. 
Como ação de melhoria definitiva: o fornecedor detectou que a causa raiz do problema, estava no processo de armazenamento do componente no casulo do carretel. Para corrigir definitivamente o problema, foi feita uma alteração nas dimensões internas do casulo utilizado. A Figura 7 mostra a ação corretiva retirada da resposta oficial do fornecedor:

Figura 7 - Ação corretiva retirada da resposta oficial do fornecedor

\section{Corrective Action:}

\section{Permanente action:}

1. Change flange construction of carrier and wide flange to both left and right sides, so that to avoid damaging lead when put products into carrier.

D/C for adopt improved CARRIER: 2012.02.22 Responsible person: Justin

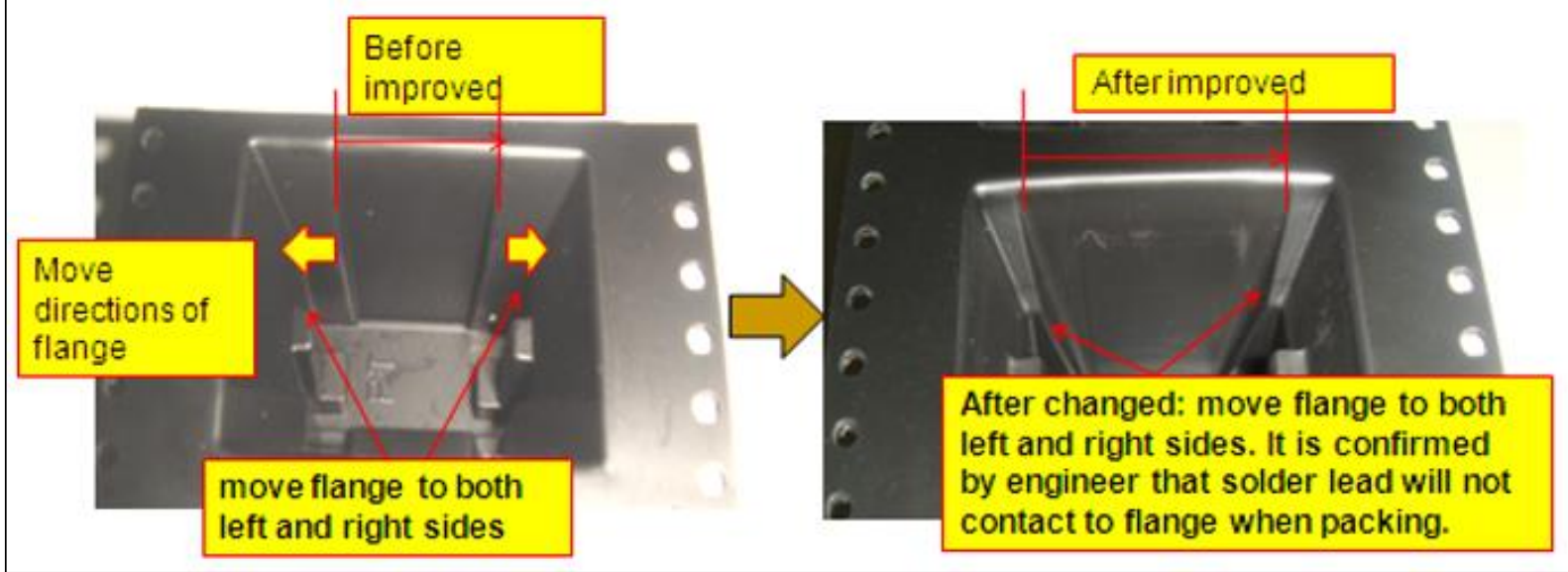

Fonte: Fornecedor da empresa estudada.

\subsection{Fase 5: Controlar}

Os lotes corrigidos começaram a ser usados na linha a partir da semana 23, quando o problema foi eliminado definitivamente em toda a cadeia, ou seja, na fase SMT - origem (Figura 8), na fase de inserção manual - cliente interno (Figura 9) e na fase de montagem do produto acabado - cliente externo (Figura 10). 
Figura 8 - Defeitos na origem

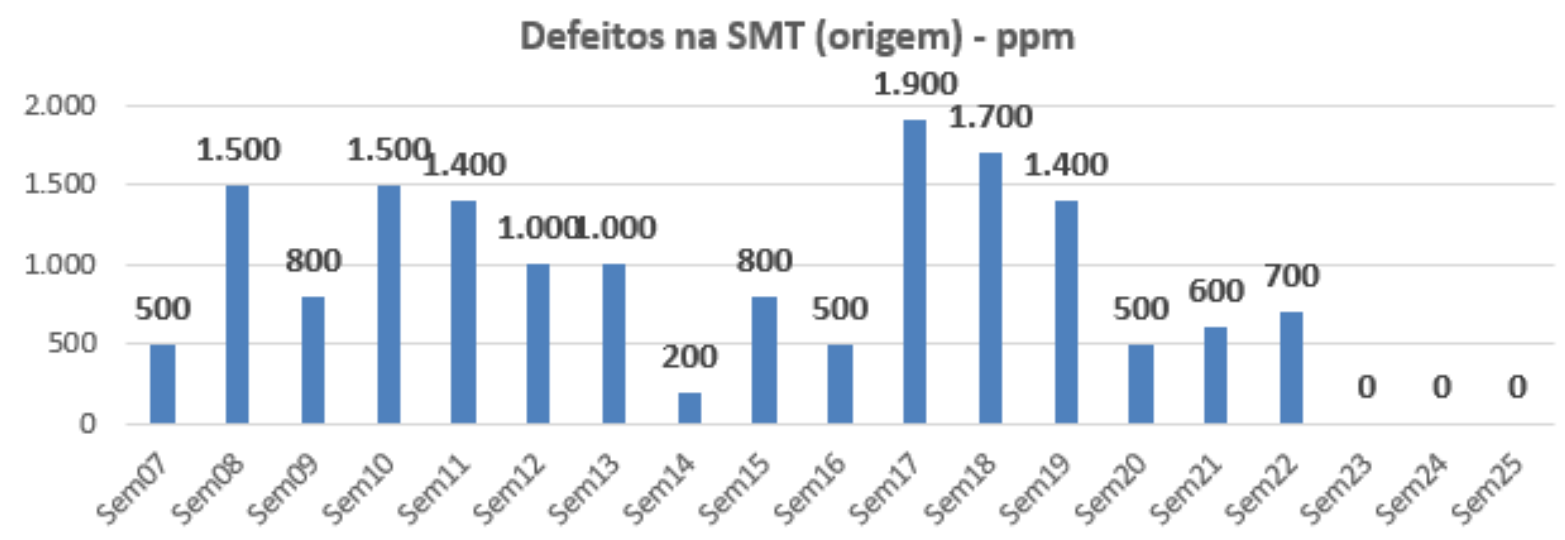

Fonte: Autores.

Figura 9 - Defeitos no cliente interno

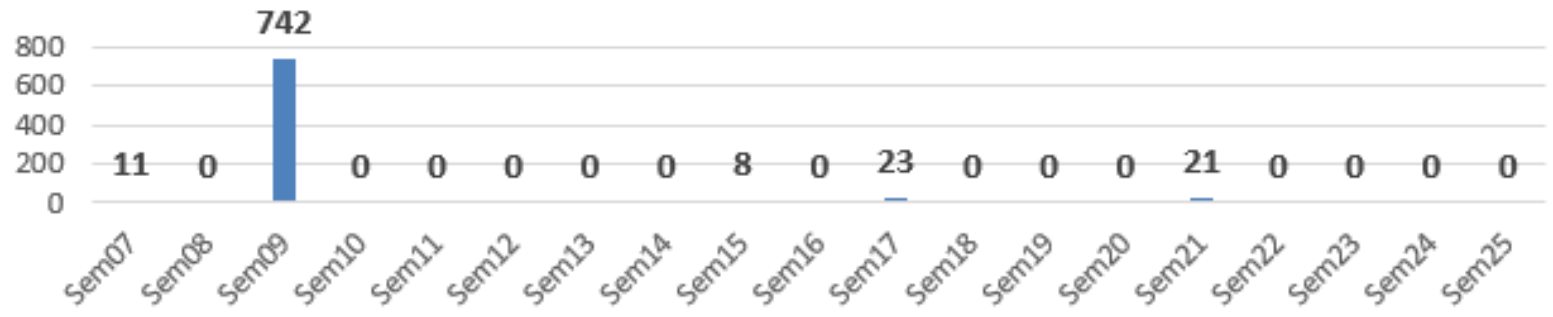

Fonte: Autores.

Figura 10 - Defeitos no cliente externo

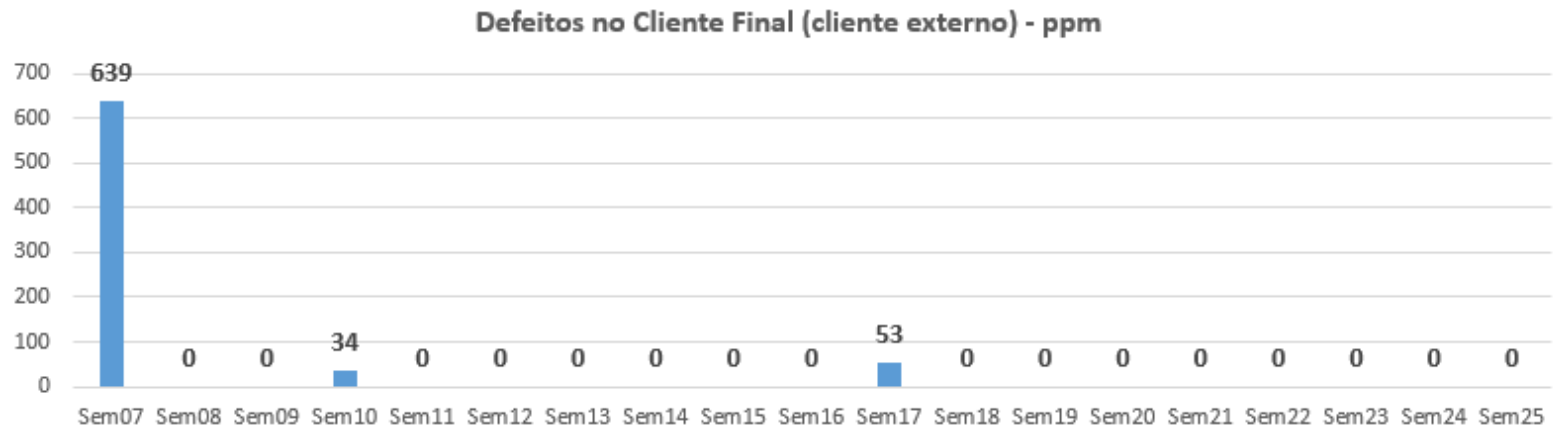

Fonte: Autores.

A fim de manter o processo sob controle, todas as documentações FMEA, Control Plan e Instruções de Trabalho foram atualizadas.

Impactos no valor da organização: satisfação do cliente, a ferramenta Jidoka foi disseminada, zero defeito causado por problema de coplanaridade do HDMI, impacto moral nos funcionários, ganho financeiro anual de $\$ 5.352$. 


\section{Discussões}

Algumas pesquisas têm abordado o Jidoka, mas este tema não é facilmente encontrado na literatura.

As pesquisas de Araujo e Costa (2012), Araujo (2012) estudaram um tipo de negócio completamente diferente do ambiente dessa pesquisa. O estudo de (Ferreira \& Ciuccio, 2012) tiveram seu foco principal no aumento de produtividade enquanto o foco desta pesquisa foi "zero defeito" por meio da aplicação do Jidoka. Ainda que independentemente do tipo de foco, os indicadores secundários também experimentam impactos positivos em seu desempenho.

Apesar do estudo de Silva e Santos (2010) também ter sido aplicado em um processo de SMT, no qual ele faz uma análise crítica da presença dos conceitos de Jidoka nas máquinas usadas por esse tipo de linha de montagem, a pesquisa aqui apresentada mostra que também neste tipo de processo ocorrem defeitos onde as máquinas instaladas não são capazes de detectar ou corrigir o problema antes que ele ocorra, sendo altamente pertinente a aplicação do Jidoka para melhorar continuamente este tipo de processo, atingindo zero defeito do problema reclamado pelo cliente.

Os resultados dessa pesquisa apontam que a aplicação de Jidoka, mesmo em máquinas de SMT, as quais normalmente já são projetadas com esse conceito, é eficaz para resolver falhas reais em uma indústria real, possibilitando ganho financeiro, melhoria da qualidade e impacto moral positivo entre os funcionários.

\section{Conclusão}

Este estudo responde à questão sobre outra maneira de aplicação da ferramenta Jidoka em processos de SMT, pois mesmo que este já tenha o conceito Jidoka intrínseco desde de sua concepção, é possível estabelecer nova prática para eliminação de falhas.

No ramo teórico, esta pesquisa apresenta um estudo de caso em que se aplicam importantes conceitos e ferramentas relacionadas a Engenharia de Produção. A metodologia aplicada foi eficaz e mostra como toda a filosofia enxuta do Lean Manufacturing pode ser utilizada em conjunto com o método de melhoria contínua do Six Sigma (bem como suas ferramentas, a exemplo do DMAIC que foi usado no estudo), além de fornecer um framework detalhado para sua implementação.

Na prática, esta pesquisa contribuiu para a satisfação do cliente com eliminação do defeito de coplanaridade do conector HDMI, para a redução dos custos de reprocesso (\$5.352), além do impacto moral em todos os membros do time. Este efeito foi mantido pela atualização dos documentos pertinentes, assim como pela manutenção dos equipamentos. Esta foi primeira aplicação do Jidoka na fábrica estudada, onde, devido ao sucesso do projeto, foi rapidamente disseminado entre os colaboradores por todas as linhas instaladas. Essa divulgação contribui para multiplicação do estudo, sendo relevante para aplicação em diversas outras organizações industriais.

Como recomendações para trabalhos futuros, ficam as seguintes sugestões: 
- Aplicação dessa mesma metodologia para outros tipos de processos. No caso de fabricação de placas é possível estender essa pesquisa para o processo de montagem Through Hole Technology (THT) e para montagem na inserção manual. Podendo também ser aplicado para processo de montagem de bem final. Mas, nada impede, de aplicar o mesmo método para outros tipos de indústrias (metalurgia, injeção plástica e outros);

- Nesse estudo, a ferramenta DMAIC foi escolhida, mas existem muitas outras ferramentas que também podem ser utilizadas.

\section{Referências}

Abdulmouti, H. (2015). The role of Kaizen (continuous improvement) in improving companies' performance: A case study. Dans 5th International Conference on Industrial Engineering and Operations Management (IEOM) (p. 6). Dubai.

Al-bahi, A. M., \& Soliman, A. Y. (2018). A Six-Sigma Approach to Improve the Acquisition of Engineering Competencies, 1561-1568. https://doi.org/10.1109/EDUCON.2018.8363418

Alvarez, J. C. (2015). Lean design for Six Sigma: An integrated approach to achieving product reliability and low-cost manufacturing. International Journal of Quality and Reliability Management, 32(8), 895-905. https://doi.org/10.1108/IJQRM-08-2012-0125

Araujo, F. J. (2012). Aplicação da Autonomação Como Estratégia De Otimização De Uma Farmácia Periférica: Estudo De Caso Em Um Hospital de Grande Porte. Dans XXXII Encontro Nacional de Engenharia de Produção (p. 11). Bento Gonçalves.

Araujo, F. J., \& Costa, C. L. de A. (2012). Aplicação da autonomação como estratégia de otimização de uma farmácia periférica: estudo de caso em um hospital de grande porte. Revista de Gestão em Sistemas de Saúde., 1(2), 86-101.

Audenino, A. (2012). Kaizen and Lean management autonomy and self-orientation, potentiality and reality... Dans 2nd International Conference on Communications, Computing and Control Applications (CCCA) (pp. 1-6). Marseilles.

Bove, T. C. M., \& Gerber, J. Z. (2013). Proposta de melhorias aos impactos causados por downtimes numa fábrica de montagem de computadores. Dans XXXIII Encontro Nacional de Engenharia de Produção (p. 14). Salvador.

Chen, M., \& Lyu, J. (2009). A Lean Six-Sigma approach to touch panel quality improvement. Production Planning and Control, 20(5), 445-454. https://doi.org/10.1080/09537280902946343

Dahlgaard, J. J., \& Dahlgaard-Park, S. M. (2006). Lean production, six sigma quality, TQM and company culture. TQM Magazine, 18(3), 263-281. https://doi.org/10.1108/09544780610659998

Ferreira, P. G., \& Ciuccio, R. L. (2012). Princípios do Lean Manufacturing: A Autonomação como Ferramenta para o Aumento de Produtividade e Competitividade na Indústria. Dans XIX Simpósio de Engenharia de Produção (pp. 1-8). Bauru.

Gonçalves, W. K. F. (2009). Utilização de Técnicas Lean e Just in Time na Gestão de Empreendimentos e Obras. IFT.

Grout, J. R., \& Toussaint, J. S. (2010). Mistake-proofing healthcare: Why stopping processes may be a good start. Business Horizons, 53(2), 149-156.

Guimaraes, L. da S., Colares, C. B., Medeiros, H. da S., \& Santana, A. F. B. (2014). Lean Manufacturing Na Industria De Componentes De Refrigeração. Dans XXXIV Encontro Nacional de Engenharia de Produção (pp. 1-17). Curitiba.

Holweg, M. (2007). The genealogy of Lean Production. journal of Operations Management, 5(6), 420-437.

Iwao, S. (2017). Revisiting the existing notion of continuous improvement (Kaizen): literature review and field research of Toyota from a perspective of innovation. Evolutionary and Institutional Economics Review, 14(1), 29-59.

Jasti, N. V. K., \& Kodali, R. (2015). Lean production: literature review and trends. International Journal 
of Production Research, 53(3), 1-19.

Junior, M. L., \& Calarge, F. A. (2013). A Abordagem Do Lean Six Sigma No Desenvolvimento De Fornecedores : Uma Análise No Setor de Eletrodomésticos. Dans XXXIII Encontro Nacional de Engenharia de ProduçãoEncontro Nacional de Engenharia de Produção (p. 16). Salvador, BA.

Kumar, M., Antony, J., Singh, R. K., Tiwari, M. K., \& Perry, D. (2006). Implementing the lean sigma framework in an Indian SME: A case study. Production Planning and Control, 17(4), 407-423. https://doi.org/10.1080/09537280500483350

Li, J., \& Blumenfeld, D. (2007). Quantitative analysis of a transfer production line with Andon. IIE Transactions (Institute of Industrial Engineers), 38(10), 837-846.

Moura, W. de M. (2014). Indicador Remoto de Falhas - Ferramenta de Auxílio à Autonomação. Dans XXXIV Encontro Nacional de Engenharia de Produção (p. 21). Curitiba.

Nunes, F., Vaccaro, G. L. R., \& Antunes Júnior, J. A. V. (2017). The development of the Hyundai Production System: The historical evolution. Journal of Manufacturing Systems, 43(1), 47-57.

Ohno, T. (1988). Toyota Production System: Beyond Large-Scale Production. Tokio : Productivity, INC.

Oliveira, D. L. de, Santos, P. P. P., Mazzuco, D. E., \& Frazzon, E. M. (2017). Regras de Despacho Aplicadas ao Problema do Roteamento de Veículos: Uma comparação Via Simulação. Dans XXXVII Encontro Nacional de Engenharia de Produção (p. 14). Joinville.

PYZDEK, T., \& KELLER, P. A. (2014). The Six Sigma handbook. Search. . : McGraw-Hill Education.

Radnor, Z. J., Holweg, M., \& Waring, J. (2012). Lean in healthcare: The unfilled promise? Social Science and Medicine, 74(3), 364-371.

Shingo, S. (1986). Zero Quality Control: Source inspection and the poka-yoke system. Cambridge : Productivity, Inc.

Silva, M. G. da, \& Santos, A. R. dos. (2010). Conceitos E Práticas Da Autonomação Em Uma Empresa Eletrônica Brasileira : Um Estudo de Caso. Dans XXX Encontro Nacional de Engenharia de Produção (p. 12). São Carlos.

Soliman, M., \& Saurin, T. A. (2017). Lean production in complex socio-technical systems: A systematic literature review. Journal of Manufacturing Systems, 45(1), 135-148.

Tsuda, Y. (1993). Implications of foolproofing in the manufacturing process. Quality through engineering design, 1(1), 79-95.

UhImann, I. R. (2015). Aplicação De Ferramentas do Lean Manufacturing em um Processo de SMT: Estudo de Caso. Universidade Federal do Pará.

UhImann, I. R., Silva, C. A. de S., Oliveira, D. L. de, \& Frazzon, E. M. (2017). Proposta de Manutenção Produtiva Total (TPM) em uma Máquina Impressora de Pasta de Solda em um Processo SMT:

Estudo de Caso. Dans XXXVII Encontro Nacional de Engenharia de Produção (p. 26). Joinville.

Vinodh, S., Kumar, S. V., \& Vimal, K. E. K. (2014). Implementing lean sigma in an Indian rotary switches manufacturing organisation. Production Planning and Control, 25(4), 288-302. https://doi.org/10.1080/09537287.2012.684726

Voss, C., Tsikriktsis, N., \& Frohlich, M. (2002). Case research in operations management. International Journal of Operations \& Production Management, 22(2), 195-219.

Wang, F. K., \& Chen, K. S. (2010). Applying Lean Six Sigma and TRIZ methodology in banking services. Total Quality Management and Business Excellence, 21(3), 301-315. https://doi.org/10.1080/14783360903553248

Wei, C., \& Cheng, Y. (2018). Six Sigma project selection using fuzzy multiple attribute decision-making method. Total Quality Management, O(0), 1-24. https://doi.org/10.1080/14783363.2018.1473029

Womack, J. P., Jones, D. T., \& Roos, D. (2007). The Machine that Changed the World (3 éd.). New York: Simon and schuster.

Yin, R. K. (2015). Estudo de caso: planejamento e método. Porto Alegre : Bookman.

Youssouf, A., Rachid, C., \& Ion, V. (2014). Contribution to the Optimization of Strategy of Maintenance by Lean Six Sigma. Physics Procedia, 55(1), 512-518.

Recebido em: 14 mar. 2018 / Aprovado em: 29 jun. 2018

Para referenciar este texto

American Psychological Association (APA)

Uhlmann, I. R., Silva, C. A. de S., Quadras, D. L. de O., \& Frazzon, E. M. (2020).

Aplicação do Jidoka em um processo SMT: estudo de caso. Exacta, 18(3), 459-

474. https://doi.org/10.5585/ExactaEP.v18n3.8473. 